\title{
Grading the intensity of nondental orofacial pain: identification of cutoff points for mild, moderate, and severe pain
}

\author{
This article was published in the following Dove Press journal: \\ Journal of Pain Research \\ 20 February 2015 \\ Number of times this article has been viewed
}

\author{
Vlaho Brailo' \\ Joanna M Zakrzewska ${ }^{2}$ \\ 'Department of Oral Medicine, School \\ of Dental Medicine, University of \\ Zagreb, Zagreb, Croatia; ${ }^{2}$ Facial Pain \\ Unit, Division of Diagnostic, Surgical \\ and Medical Sciences, Eastman Dental \\ Hospital, UCLH NHS Foundation \\ Trust/University College London, \\ London, UK
}

Background: When assessing pain in clinical practice, clinicians often label pain as mild, moderate, and severe. However, these categories are not distinctly defined, and are often used arbitrarily. Instruments for pain assessment use more sophisticated scales, such as a 0-10 numerical rating scale, and apart from pain intensity assess pain-related interference and disability. The aim of the study was to identify cutoff points for mild, moderate, and severe nondental orofacial pain using a numerical rating scale, a pain-related interference scale, and a disability measurement.

Materials and methods: A total of 245 patients referred to the Facial Pain Unit in London were included in the study. Intensity and pain-related interference were assessed by the Brief Pain Inventory. Pain-related disability was assessed by the Chronic Graded Pain Scale. Average pain intensity (0-10) was classified into nine schemes with varying cutoff points of mild, moderate, and severe pain. The scheme with the most significant intergroup difference, expressed by multivariate analysis of variance, provided the cutoffs between mild, moderate, and severe pain.

Results: The combination that showed the greatest intergroup differences for all patients was scheme 47 (mild 1-4, moderate 5-7, severe 8-10). The same combination provided the greatest intergroup differences in subgroups of patients with temporomandibular disorder and chronic idiopathic facial pain, respectively. Among the trigeminal neuralgia patients alone, the combination with the highest intergroup differences was scheme 48 (mild 1-4, moderate 5-8, severe 9-10).

Conclusion: The cutoff points established in this study can discriminate in pain intensity categories reasonably well, and showed a significant difference in most of the outcome measures used.

Keywords: chronic orofacial pain, cutoff point, trigeminal neuralgia, temporomandibular disorder

\section{Introduction}

After the correct diagnosis has been established, a vital step for effective treatment of chronic pain is assessment of its intensity and impact. Clinicians most often use categories like mild, moderate, and severe. The categories are not distinctly defined, and are often used arbitrarily. More sophisticated tools like the Brief Pain Inventory (BPI) or the Chronic Graded Pain Scale ${ }^{1,2}$ most often use a 0-10 numeric rating scale (NRS). Apart from pain intensity, these instruments assess the degree of pain-related functional interference and its impact on quality of life. In spite of the availability of these instruments, many clinicians prefer the use of the mild, moderate, and severe categories.

The question, therefore, arises if it is possible to define more precisely categories like mild, moderate, and severe using these instruments. An NRS cannot just be divided
Correspondence: Vlaho Brailo

Department of Oral Medicine, School of Dental Medicine, University of Zagreb,

5 Gundulićeva Street, Zagreb 10000 ,

Croatia

Tel +385 I 4802 I74

Fax +385 । 4830819

Email brailo@sfzg.hr 
into three equal parts, because the relationship between pain intensity and functional interference is not linear. The reduction in the same interval of intensity on different parts of an NRS does not produce similar reduction in functional impairment or patient's general well-being. ${ }^{3}$ The relationship between pain intensity and pain-related interference would thus be more correctly defined by identifying cutoff points for mild, moderate, and severe pain.

This method, introduced by Serlin et al, ${ }^{3}$ has been widely applied. Cutoff points have been determined for various chronic pain conditions, such as cancer pain, diabetic neuropathy, osteoarthritis, low-back pain, phantom limb pain, neck pain, and musculoskeletal pain. ${ }^{4-10}$ In these studies, cutoff points for mild pain ranged from 2 to 5 , while cutoff points for severe pain ranged from 6 to 8 . The results of these studies suggest that the pain experience and pain-related functional impairment depends on the condition and the affected site. However, cutoff points for nondental orofacial pain have not been identified. Identifying cutoff points for mild, moderate, and severe nondental orofacial pain would aid clinicians and investigators in defining a measurable target range of adequate pain relief. The severity and impact of the different orofacial pains is large, especially if rare conditions, such as trigeminal neuralgia (TN) and the trigeminal autonomic cephalalgias, are included. ${ }^{11-13}$ The cutoff points could also be used to determine entry into clinical trials.

The aim of this study was to identify cutoff points between mild, moderate, and severe non dental orofacial pain, to assess if the cutoff points can be generally applied to all nondental orofacial pain conditions or if they are specific to each of the disorders, and to determine if the cutoff points discriminate between three intensity categories in several patient-related outcome measures (functional impairment, pain-related disability, depression, and anxiety).

\section{Materials and methods}

The study was reviewed and approved by the European Association of Oral Medicine board. All patients referred to the national Facial Pain Unit in London during a 6-week period in 2009 were asked to participate in the study. The Facial Pain Unit sees 700 new patients and 1,400 follow-up patients per year, and all patients have previously seen at least one primary care provider.

Criteria for referral to the unit are chronic pain, ie, over 3 months and exclusion of dental causes. Therefore, a 6-week period was assumed to provide a representative sample of non dental orofacial pain patients in terms of demographics and diagnosis.
The inclusion criteria were presence of nondental orofacial pain for more than 3 months, age over 18 years, and ability to understand questionnaires (one of the essential requirements for assessment in the clinic is the completion of several questionnaires that assess patients' treatment expectations, sociodemographic data, pain intensity, pain-related interference, and pain-related disability). Theoretically, patients who did not understand the questionnaires would have been excluded from the study, but this did not occur, as the questionnaires were sent out ahead of the appointment and patients were encouraged to ask for help if they had difficulties completing them. Fifteen however did not complete all the questions, and so were excluded.

Demographic and clinical data (age, sex, duration of pain, and number of specialists seen before referral to the Facial Pain Unit) were registered. Intensity and pain-related functional impairment were assessed by the BPI. ${ }^{1}$ The BPI uses an eleven-item NRS (0-10), where patients rate their worst, least, average, and current pain intensity, as well as interference with various aspects of everyday life (general activity, mood, walking, work, relationships with other people, sleep, and enjoyment in life). Pain-related disability was assessed by the Chronic Graded Pain Scale. ${ }^{2}$ This classifies patients into four disability categories (1, low intensity, low disability; 2, high intensity, low disability; 3 , high disability, moderately limiting; and 4, high disability, severely limiting) based on interference with everyday activity and period of limited activity because of pain. The Hospital Anxiety and Depression Scale (HADS) was used to assess anxiety and depression. ${ }^{14}$ According to the HADS score, patients were classified as follows: 0-7, no depression/anxiety; 8-10, borderline depression/anxiety; and 11 and over, depression/anxiety.

Patients were classified into three subgroups according to their diagnosis: all types of $\mathrm{TN}$, myogenic temporomandibular disorder (TMD) and persistent/chronic idiopathic facial pain (CIFP). TN was diagnosed according to the International Classification of Headache Disorders criteria. ${ }^{15}$ The diagnosis of TMD was made according to the following criteria: 1) pain and tenderness of the muscles of mastication of 3 months' duration or longer and 2) no clinical and/ or radiographic evidence of organic temporomandibular joint disorder. ${ }^{16}$ The third group was heterogeneous, and consisted of patients who did not fit into the previous two groups (patients with CIFP, burning mouth syndrome, and posttraumatic neuropathic pain). The diagnoses of these were made according to the International Classification of Headache Disorders criteria. ${ }^{15}$ 


\section{Statistical analysis}

SPSS software (version 20 for Windows; IBM, USA) was used for data analysis. Depending on the distribution of the data, mean and standard deviation or median and range were used to summarize the data. For categorical variables, differences between groups were tested by the $\chi^{2}$ test. For numerical variables, group differences were assessed by one-way analysis of variance (ANOVA), followed by post hoc Bonferroni test or the Kruskal-Wallis test followed by Mann-Whitney $U$ tests, with $P$-values adjusted for multiple testing if the assumptions of the ANOVA were not satisfied.

Determination of cutoff points was performed as described by Serlin et al. ${ }^{3}$ Average pain intensity was classified into nine schemes with different cutoff points of mild, moderate and severe pain: 1 , scheme 35 (mild 1-3, moderate 4-5, severe 6-10); 2, scheme 36 (mild 1-3, moderate 4-6, severe 7-10); 3 , scheme 37 (mild 1-3, moderate 4-7, severe 8-10); 4, scheme 38 (mild 1-3, moderate 4-8, severe 9-10); 5, scheme 46 (mild 1-4, moderate 5-6, severe 7-10); 6, scheme 47 (mild 1-4, moderate 5-7, severe 8-10); 7, scheme 48 (mild 1-4, moderate 5-8, severe 9-10); 8, scheme 57 (mild 1-5, moderate 6-7, severe 8-10); and 9, scheme 58 (mild 1-5, moderate 6-8, severe 9-10). Nine multivariate one-way ANOVAs were performed, with the intensity group (mild, moderate, and severe) as the independent variable and seven pain-interference domains from the BPI as the dependent variable. The scheme with the most significant intergroup difference, expressed by the smallest $P$-value determined from Wilks' lambda, was considered to indicate the maximum difference between the groups, and thus provided the cutoffs between mild, moderate, and severe pain.

To assess if the determined cutoff points discriminated adequately between pain-intensity categories, patients were compared on various outcome measures using one-way ANOVAs, followed by Bonferroni post hoc comparisons where appropriate (for pain-related interference), or $\chi^{2}$ tests (for pain-related disability, depression, and anxiety). In all analyses, $P<0.01$ was considered statistically significant. This significance level was chosen rather than the conventional 0.05 level to avoid spuriously significant results arising from multiple testing. Effect size was expressed by $\eta^{2}$ or $\varphi$-coefficient where appropriate.

\section{Results}

\section{Demographic and clinical characteristics of the participants}

A total of 245 patients were included in the study. There were $186(76 \%)$ female patients and $59(24 \%)$ male patients.
A total of 112 patients had TMD, 85 patients had CIFP, and 48 patients had TN. The median age of the participants was 47 (range 18-84) years. The demographic and clinical data of the patients are shown in Table 1.

No significant difference in sex was observed between the three groups of patients. A significant difference in median age was observed between the groups: TN patients were significantly older than CIFP and TMD patients $(P=0.002$ and $P<0.001$, respectively). CIFP patients were on average significantly older than patients with TMD $(P<0.001)$. Median duration of pain was significantly longer in $\mathrm{TN}$ patients compared to TMD and CIFP patients $(P<0.001$ and $P=0.027$, respectively). TN and CIFP patients visited significantly more pain specialists before referral to the Facial Pain Unit than TMD patients $(P=0.004$ and $P<0.001$, respectively). No significant difference in the proportion of patients with anxiety and depression was found between the three groups of participants.

The mean value of the average pain intensity was significantly higher in CIFP patients than in TMD patients $(P=0.003)$. No significant differences in the mean values of average pain intensity were observed between TMD and TN patients or CIFP and TN patients $(P=0.999$ and $P=0.186$, respectively). No significant differences were observed in the mean worst, least, and current pain-intensity scores between three groups of participants $(P=0.081, P=0.025$, and $P=0.097$, respectively). No significant differences in pain-related interference were observed between the three groups of participants $(P=0.058)$. No significant differences in pain-related disability were observed between the three groups of participants $(P=0.206)$.

\section{Determination of cutoff points}

Cut-off points were determined as described in the Materials and methods section. The combination that showed the greatest intergroup differences for all patients was scheme 47 (mild 1-4, moderate 5-7, severe 8-10). The same combination provided the greatest intergroup differences in TMD and CIFP patients. Among the TN patients alone, the combination with the highest intergroup differences was scheme 48 (mild 1-4, moderate 5-8, severe 9-10) (Table 2). No significant difference in cutoff points between males and females was observed. ( $\eta^{2}$ ranged from $0.35-0.41$, indicating strong effect).

\section{Assessment of cutoff points}

As explained in the Materials and methods section, in order to assess if the optimal cutoff points discriminated between 
Table I Demographic and clinical data of the patients

\begin{tabular}{|c|c|c|c|c|c|}
\hline & Total & TMD & CIFP & TN & $\mathbf{P}$ \\
\hline Sex, n (\%) & & & & & $\chi^{2}$ \\
\hline Female & $186(76 \%)$ & $90(80.4 \%)$ & $63(74.1 \%)$ & $33(70.2 \%)$ & 0.333 \\
\hline Male & $59(24 \%)$ & $22(19.6 \%)$ & $22(25.9 \%)$ & $15(29.8 \%)$ & \\
\hline \multirow[t]{2}{*}{ Total } & 245 & 112 & 85 & 48 & \\
\hline & & & & & Mann-Whitney U \\
\hline Age, years, median (range) & $47(12-84)$ & $36.5(12-77)$ & $50(23-84)$ & $64(20-8 I)$ & $<0.00 I^{*}$ \\
\hline Duration of pain, months, median (range) & $27(6-468)$ & $24(6-180)$ & $36(6-360)$ & $64(6-468)$ & $<0.00 I^{*}$ \\
\hline Referred by, n (\%) & & & & & $\chi^{2}$ \\
\hline GDP & $7 \mid(41 \%)$ & $37(52.1 \%)$ & $27(37.5 \%)$ & $7(23.3 \%)$ & 0.051 \\
\hline GMP & $43(24.9 \%)$ & $18(25.4 \%)$ & $15(20.8 \%)$ & $10(33.3 \%)$ & \\
\hline \multirow[t]{2}{*}{ Specialist } & $59(34.1 \%)$ & $16(22.5 \%)$ & $30(4 I .7 \%)$ & $13(43.3 \%)$ & \\
\hline & & & & & Mann-Whitney U \\
\hline $\begin{array}{l}\text { Number of specialists visited before the } \\
\text { referral, median (range) }\end{array}$ & $2(0-7)$ & I (0-6) & $2(0-7)$ & $2(0-5)$ & $<0.00 I^{*}$ \\
\hline Depression, n (\%) & & & & & $x^{2}$ \\
\hline None & $60(52.2 \%)$ & $4 \mathrm{I}(67.2 \%)$ & $15(57.7 \%)$ & $19(67.9 \%)$ & 0.140 \\
\hline Borderline/mild depression & $25(21.7 \%)$ & $12(19.7 \%)$ & $3(11.5 \%)$ & $7(25 \%)$ & \\
\hline Severe depression & $30(26.1 \%)$ & $8(13.1 \%)$ & $8(30.8 \%)$ & $2(7.1 \%)$ & \\
\hline Anxiety, n (\%) & & & & & $x^{2}$ \\
\hline None & $75(65.2 \%)$ & $27(44.3 \%)$ & $16(61.5 \%)$ & $17(60.7 \%)$ & 0.493 \\
\hline Borderline/mild anxiety & $22(19.1 \%)$ & $16(26.2 \%)$ & $4(15.4 \%)$ & $5(17.9 \%)$ & \\
\hline Severe anxiety & $18(15.7 \%)$ & 18 (29.5\%) & $6(23.1 \%)$ & $6(21.4 \%)$ & \\
\hline Pain intensity, mean \pm SD & & & & & Analysis of variance \\
\hline Average & $4.9 \pm 2.9$ & $4.3 \pm 2.7$ & $5.7 \pm 2.8$ & 5.2. \pm 3.2 & $0.004^{*}$ \\
\hline Worst & $5.7 \pm 3.2$ & $5.2 \pm 3.2$ & $6.2 \pm 3$ & $6 \pm 3.4$ & 0.081 \\
\hline Least & $3.5 \pm 3.1$ & $3 \pm 2.9$ & $4.2 \pm 3.1$ & $3.8 \pm 3.6$ & 0.025 \\
\hline Current & $4.1 \pm 3.2$ & $3.7 \pm 3.1$ & $4.7 \pm 3.1$ & $4 \pm 3.5$ & 0.097 \\
\hline Pain-related interference, mean \pm SD & $3.7 \pm 2.9$ & $3.3 \pm 2.8$ & $4.3 \pm 3$ & $3.8 \pm 3$ & 0.055 \\
\hline Pain-related disability, n (\%) & & & & & $x^{2}$ \\
\hline Grade I & $64(28.1 \%)$ & $38(36.9 \%)$ & $16(21.3 \%)$ & $10(20 \%)$ & 0.206 \\
\hline Grade 2 & $62(27.2 \%)$ & $28(27.2 \%)$ & $22(29.3 \%)$ & $12(24 \%)$ & \\
\hline Grade 3 & 35 (I5.4\%) & $14(13.6 \%)$ & $14(18.7 \%)$ & $7(14 \%)$ & \\
\hline Grade 4 & $67(29.3 \%)$ & $23(22.3 \%)$ & $23(30.7 \%)$ & $21(42 \%)$ & \\
\hline
\end{tabular}

Notes: $* P<0.01$. Specialist - health care professional with dental or medical specialization; number of specialists visited before the referral, number of medical and/or dental specialists that patient saw before he/she was referred to the Facial Pain Unit; pain intensity (average, worst, least, right now), intensity subscales of the Brief Pain Inventory; pain-related interference, overall interference calculated from the interference subscales of the Brief Pain Inventory; pain-related disability determined by the Chronic Graded Pain Scale.

Abbreviations: TMD, temporomandibular disorder; CIFP, chronic idiopathic facial pain; TN, trigeminal neuralgia; SD, standard deviation; GDP, general dental practitioner; GMP, general medical practitioner.

the pain-intensity categories, the three intensity groups were compared on various outcome measures using univariate oneway ANOVAs, followed by Bonferroni post hoc comparisons where appropriate (pain-related interference), or $\chi^{2}$ tests (pain-related disability, depression, and anxiety).

\section{Pain interference in pain-intensity groups}

In all patients, a significant difference in the means between mild-, moderate-, and severe-pain categories was found for all BPI interference scales, as well as for overall interference (Figure 1); $\eta^{2}$ ranged from 0.26 to 0.57 , indicating a strong effect. In the subgroup of TMD patients, a significant difference between mild, moderate, and severe pain was found for all BPI interference scales, as well as for overall interference.
A post hoc test did not reveal significant difference in interference with walking ability among patients with mild and moderate pain $(P=0.286) ; \eta^{2}$ ranged from 0.22 to 0.61 , indicating a moderate-to-strong effect. In the subgroup of CIFP patients, a significant difference between mild, moderate, and severe pain was found for all BPI interference scales, as well as for overall interference. A post hoc test did not reveal a significant difference in interference with work among patients with moderate and severe pain $(P=0.228)$; $\eta^{2}$ ranged from 0.24 to 0.48 , indicating moderate-to-strong effect. In the subgroup of TN patients, a significant difference between mild, moderate, and severe pain was observed for all BPI interference scales, as well as for overall interference. Post hoc tests did not reveal significant differences in inter- 


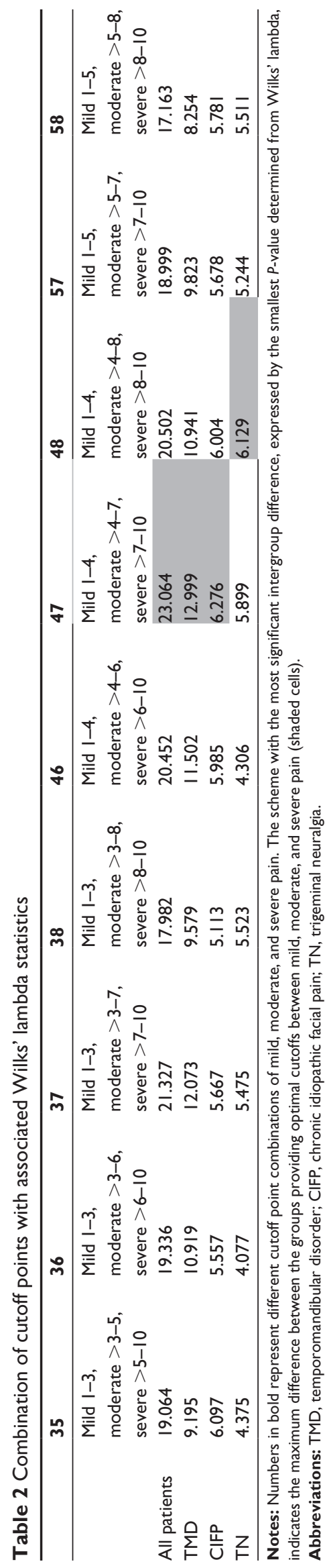

ference with mood or enjoyment of life among patients with moderate and severe pain $(P=0.786$ and $P=0.270$, respectively). Furthermore, in the TN subgroup, nonsignificant differences in interference with walking ability and sleep were found between patients with mild and moderate pain ( $P=0.366$ and $P=0.318$, respectively); $\eta^{2}$ ranged from 0.3 to 0.56 , indicating a strong effect.

\section{Pain-related disability in pain-intensity groups}

A significant association was found between intensity of pain (mild, moderate and severe pain) and pain-related disability. Statistical significance was observed in all patients, as well as in all three subgroups of patients $(P<0.001, P<0.001$, $P<0.001$, and $P=0.005$, respectively); $\varphi$-values ranged from 0.66 to 0.73 , indicating a strong effect (Figure 2).

\section{Depression and anxiety in pain-intensity groups}

A significant association was observed between pain intensity (mild, moderate, and severe pain) and anxiety $(P=0.008)$ in all patients. No significant association was observed between pain intensity and depression and $P=0.014$ in any patients. Significant differences in the percentage of anxiety and depression among patients with mild, moderate, and severe pain were found in the TMD $(P<0.001$ and $P<0.001$, respectively $)$ and TN subgroups $(P<0.001$ and $P<0.001$, respectively). No significant difference in the percentages of either anxiety or depression between patients with mild, moderate, and severe pain was observed in the CIFP subgroup ( $P=0.141$ and $P=0.175$, respectively) (Figure 3); $\varphi$-values ranged from 0.34 to 1 indicating a moderate-to-strong effect.

\section{Discussion}

The results of this study indicate that in nondental orofacial pain conditions, the reduction in equal interval of pain intensity on an NRS will not produce the same level of reduction in functional impairment. This finding confirms the nonlinear relationship between pain intensity and functional impairment, as suggested by Serlin et al. ${ }^{3}$ Using the eleven-item NRS, intensity of nondental orofacial pain can be defined as follows: mild pain $0-4$, moderate pain $>4-7$, and severe pain $>7-10$. Cutoff points established in this way provide significant differences for almost all outcome measures used in this study, which is especially emphasized in the overall pain-related interference and pain-related disability. This combination of cutoff points was found to be optimal also for phantom limb pain, peripheral diabetic neuropathy, knee 


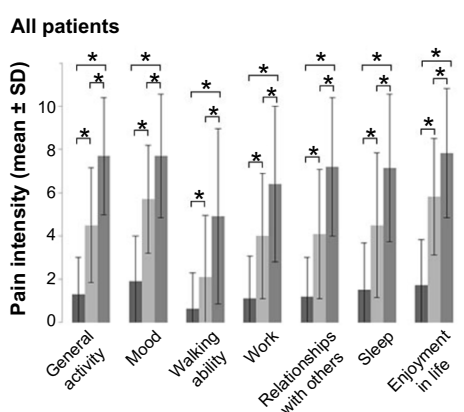

CIFP patients

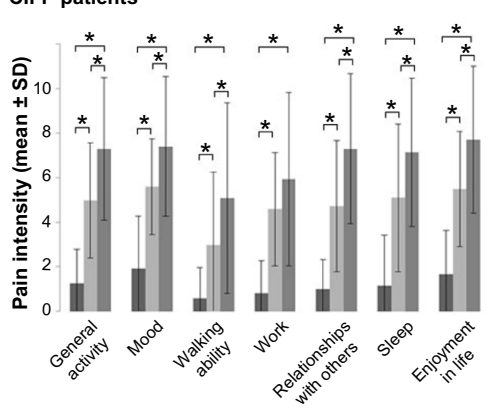

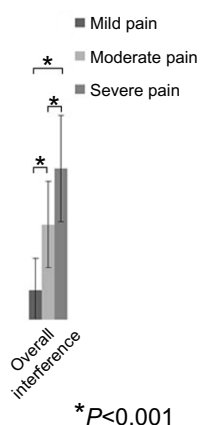

- Mild pain

$=$ Moderate pain - Severe pain

*

*
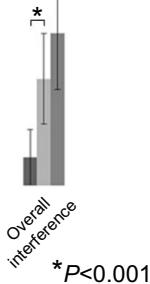



TN patients



= Mild pain

m Moderate pain

M Severe pain

*

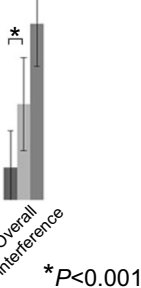

- Mild pain

= Moderate pain

= Severe pain

*

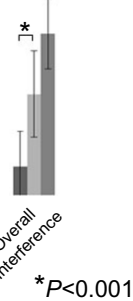

Figure I Pain-related interference in the three pain-intensity groups.

Abbreviations: TMD, temporomandibular disorder; CIFP, chronic idiopathic facial pain; TN, trigeminal neuralgia; SD, standard deviation.

osteoarthritis, and cancer pain. ${ }^{5-8}$ Chronic pain conditions like back pain, general pain, neck pain, and headache had different combinations of optimal cutoff points. ${ }^{4,6,17}$ These findings indicate that the impact of chronic pain on daily activities does not depend solely on the pain intensity, but is also dependent on the nature of the condition.

Demographic factors, such as sex and age, did not affect the established cutoff points. It is known that chronic


Figure 2 Pain-related disability in the three pain-intensity groups (grades I-4, pain-related disability determined by the Chronic Graded Pain Scale). Abbreviations: TMD, temporomandibular disorder; CIFP, chronic idiopathic facial pain; TN, trigeminal neuralgia. 
Depression-all patients
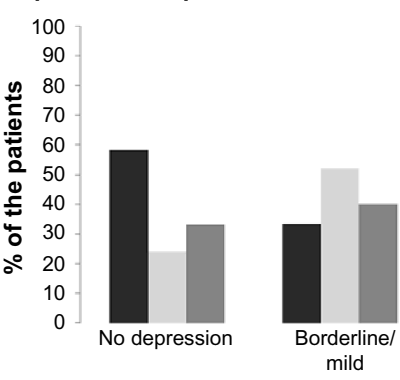

depression

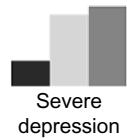

${ }^{*} P<0.001$

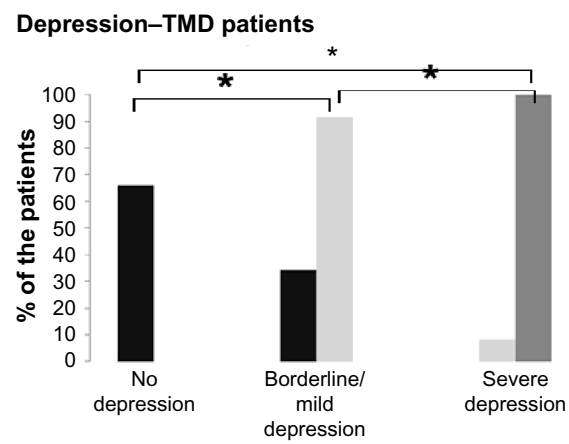

- Mild pain Moderate pain n Severe pain

Depression-CIFP patients
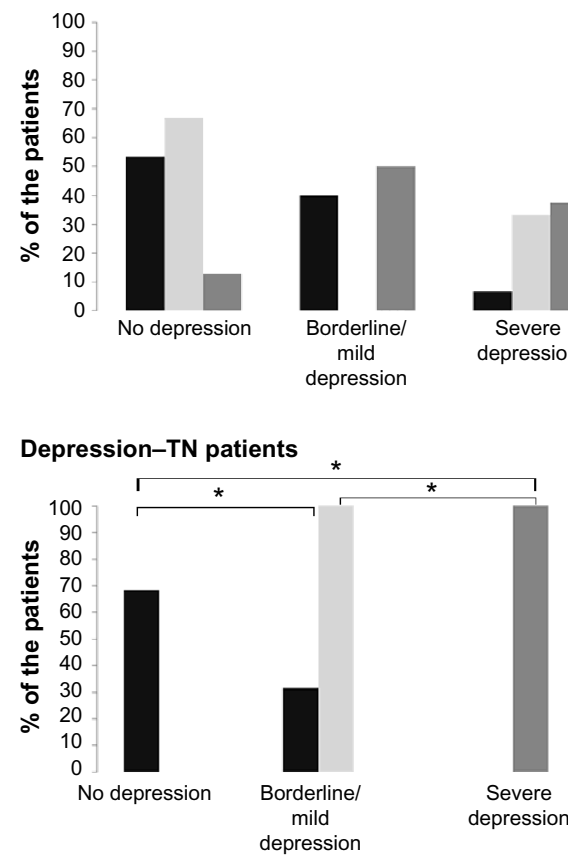

aild pain

Moderate pain

- Severe pain

\section{(1)}


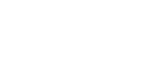

- Mild pain

Moderate pain

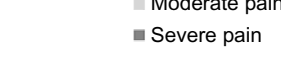

- Mild pain

a Severe pain

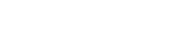

${ }^{\star} P<0.001$

Anxiety-CIFP patients



${ }^{*} P<0.001$

Moderate pain
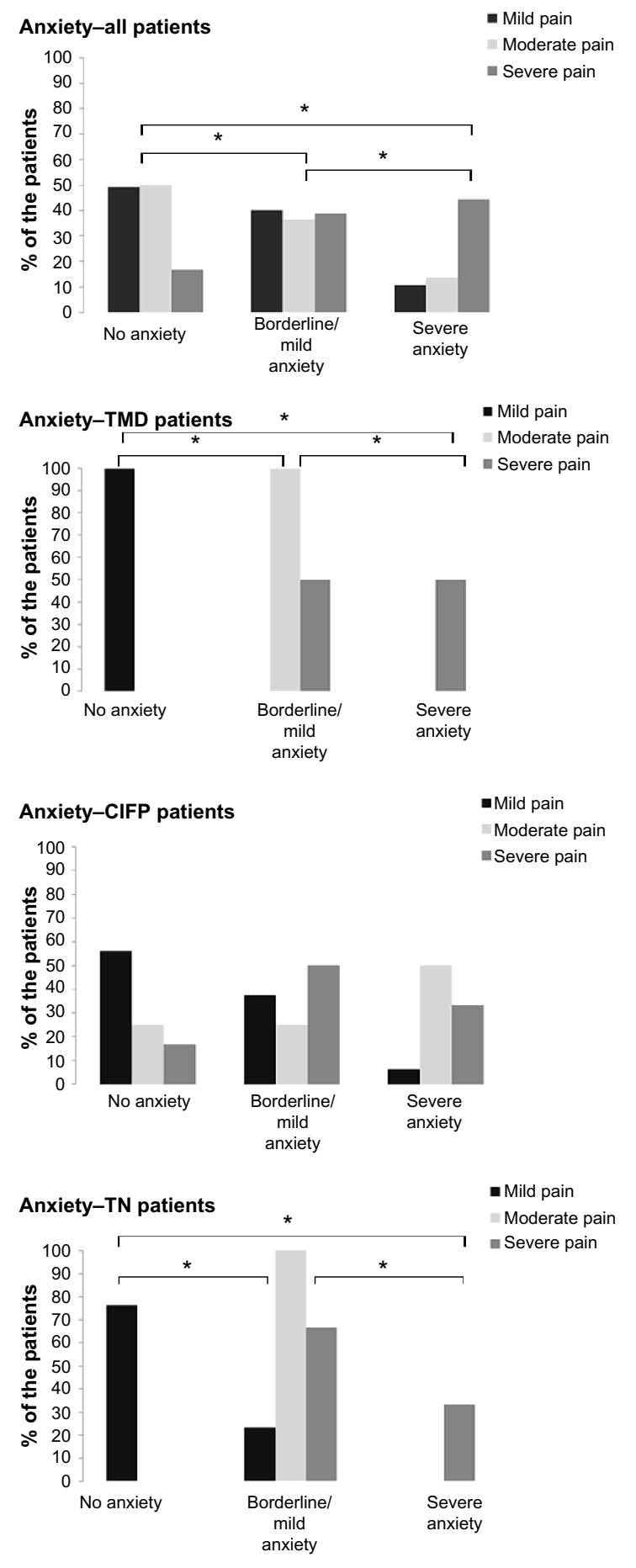

- Mild pain

Moderate pain Severe pain$$
\text { (1) }
$$

Figure 3 Anxiety and depression in the three pain-intensity groups.

Abbreviations: TMD, temporomandibular disorder; CIFP, chronic idiopathic facial pain; TN, trigeminal neuralgia.

orofacial pain conditions, such as TN, TMD, and CIFP, are more prevalent among women. Women tend to report higher pain intensity and duration of pain. ${ }^{18,19}$ However, no difference between men and women was found in any of the studies that determined cutoff points for mild, moderate, and severe pain in different chronic pain conditions. ${ }^{4-10}$ It seems that the impact of pain on everyday function diminishes sex-related 
factors, such as pain acceptance, individual coping strategies, and other risk factors. ${ }^{26-28}$ A significant difference between the three pain-intensity groups was found only for TMD and TN patients. The difference was not statistically significant in the CIFP group, which could be due to heterogeneity of the group or the aforementioned mentioned pain acceptance and individual coping strategies. Anxiety and depression were used for the comparison of intensity groups in only two studies that used the same method for the identification of cutoff points in chronic pain. ${ }^{5,8}$ Paul et al did not find significant difference in either depression or anxiety between cancer patients classified as having mild, moderate, and severe pain. ${ }^{8}$ On the other hand, in a study of Hoffman et al, anxiety and depression subscales discriminated significantly between pain-intensity subgroups in patients with diabetic neuropathy. ${ }^{5}$ The difference between the studies could have been due to the use of different scales for the assessment of depression and anxiety: Paul et al used the shortened version of the Profile of Mood States, while Hoffmann et al used the HADS. ${ }^{5,8}$ In spite of the differences, it remains important to monitor patients' psychological health, as chronic pain is a risk factor for the onset of anxiety and/or depressive disorders. $^{23-25}$

Cutoff points are not characteristic for an individual condition, but can be used in almost all nondental chronic orofacial pain conditions. The exception was TN patients, where the highest intergroup difference was obtained in scheme 48 (mild pain $1-4$, moderate $>4-8$, severe $>8-10$ ). There can be several reasons for this. First of all, the group of TN patients was very small, and the statistical analysis could have been affected by the subtle changes in the number of patients in intensity-level subgroups. On the other hand, these results could reflect the character of TN. Despite high pain intensity, the pain in $\mathrm{TN}$ is episodic, and higher pain intensity and longer duration may be needed to result in a meaningful interference with daily activities. Furthermore, in the majority of TN patients, pain can be adequately controlled with medications that can have a positive impact on pain-related interference with daily activities ${ }^{30}$ Having experienced very severe episodes of pain, patients with TN may be more discriminating about their pain severity, ie, they often distinguish between what they term "twinges" compared to "electric shocks". Unlike TN, in TMD and CIFP the pain is more or less constant and often not successfully controlled with medications that can affect patients' daily activities and quality of life. Furthermore, patients with TMD and CIFP are more likely to have other chronic pain and more psychosocial predisposing factors than TN patients, and so require a holistic treatment approach. ${ }^{31-36}$ Only $17 \%$ of the TN patients in this cohort compared to $90 \%$ of patients in the TMD and CIFP subgroups had other chronic pain including headache. This might explain why lower pain intensity can result in higher disability/functional impairment.

Classification of mild, moderate, and severe pain as defined in this study is in line with patients' definition of acceptable outcome. According to the studies of Thorne and Morley $^{37}$ and Farrar et $\mathrm{al}^{38}$ on more than 2,000 patients with various chronic pain conditions, patients' definition of "much improvement" implies reduction of 2-3 raw points or $30 \%$ on an NRS. In TMD patients, clinically important change was defined as an intensity visual analog scale score reduction of $19.5 \mathrm{~mm}$ and percentage change of $37.9 \%$ from baseline. ${ }^{39}$ Percentage change showed higher sensitivity, since raw visual analog scale score reduction was significantly affected by the baseline pain levels. ${ }^{39}$ Furthermore, the Initiative on Methods, Measurement and Pain Assessment in Clinical Trials (IMMPACT) recommends reporting on the percentages of patients achieving $\geq 30 \%$ reduction in the NRS, since this reduction appears to reflect at least moderate clinically important difference. ${ }^{40}$ Reduction of $\geq 50 \%$, on the other hand, reflects substantial improvement and should also be reported. This is especially relevant in TN, where unlike other types of nondental orofacial pain, nearly $100 \%$ pain reduction can be achieved; if not by medication, then by surgery. ${ }^{41}$

This study has several limitations that need to be addressed. According to Hirschfeld and Zernikow, the statistical method for determination of cutoff points applied in this study does not take into account the variability of the sample. ${ }^{17}$ The authors state that the differences between the groups were as a result of a chance variation rather than true differences in pain-related interference. Bootstrapping was performed, the rank ordering of the cut-off points was not affected, and the combination of 47 and 48 was still identified as the most appropriate for TMD, CIFP, and TN. The cutoff points could not represent the optimal relationship between pain intensity and functional interference in every individual patient, but for the majority of nondental orofacial pain patients, these measures are probably valid. Furthermore, one of the pain characteristics not assessed in the BPI that could be of importance is interference with eating. This characteristic would probably be emphasized in nondental chronic orofacial pain and might influence the results. An extended BPI, called BPI facial, which includes seven additional oral/facial parameters, has recently been validated in patients with TN. ${ }^{42}$ However, no data are yet available on its 
validity in other facial pain conditions. Therefore, we decided to use a validated instrument, as was used in all similar studies. ${ }^{3-10}$ Another limitation of this study is the small number of TN patients. Further studies with larger numbers of patients are therefore required.

In spite of the study limitations, we believe that the cutoff points determined in this study discriminate pain-intensity categories reasonably well and provide significant difference in most of the outcome measures used. These cutoff points would help clinicians and researchers to define more precisely satisfactory levels of pain relief in nondental orofacial pain patients. They would be of use in clinical trials and for providers of pain services when assessing pain-related outcome measures.

\section{Acknowledgments}

We are grateful to Aviva Petrie and David Boniface, who advised on the statistical analysis. VB undertook this work as part of his grant from the European Association of Oral Medicine. JMZ undertook this work at UCL/ UCLHT, who received a proportion of funding from the Department of Health's NIHR Biomedical Research Centre funding.

\section{Author contributions}

Both authors collected the data and contributed to the manuscript equally. Both authors discussed the results and commented on the manuscript.

\section{Disclosure}

The authors report no conflicts of interest in this work.

\section{References}

1. Cleeland CS, Ryan KM. Pain assessment: global use of the Brief Pain Inventory. Ann Acad Med Singapore. 1994;23(2):129-138.

2. Von Korff M, Ormel J, Keefe FJ, Dworkin SF. Grading the severity of chronic pain. Pain. 1992;50(2):133-149.

3. Serlin RC, Mendoza TR, Nakamura Y, Edwards KR, Cleeland CS. When is cancer pain mild, moderate or severe? Grading pain severity by its interference with function. Pain. 1995;61(2):277-284.

4. Fejer R, Jordan A, Hartvigsen J. Categorising the severity of neck pain: establishment of cut-points for use in clinical and epidemiological research. Pain. 2005;119(1-3):176-182.

5. Hoffman DL, Sadosky A, Dukes EM, Alvir J. How do changes in pain severity levels correspond to changes in health status and function in patients with painful diabetic peripheral neuropathy? Pain. 2010;149(2): 194-201.

6. Jensen MP, Smith DG, Ehde DM, Robinsin LR. Pain site and the effects of amputation pain: further clarification of the meaning of mild, moderate, and severe pain. Pain. 2001;91(3):317-322.

7. Kapstad H, Hanestad BR, Langeland N, Rustøen T, Stavem K. Cutpoints for mild, moderate and severe pain in patients with osteoarthritis of the hip or knee ready for joint replacement surgery. BMC Musculoskelet Disord. 2008;9:55.
8. Paul SM, Zelman DC, Smith M, Miaskowski C. Categorizing the severity of cancer pain: further exploration of the establishment of cutpoints. Pain. 2005;113(1-2):37-44.

9. Zelman DC, Dukes E, Brandenburg N, Bostrom A, Gore M. Identification of cut-points for mild, moderate and severe pain due to diabetic peripheral neuropathy. Pain. 2005;115(1-2):29-36.

10. Zelman DC, Hoffman DL, Seifeldin R, Dukes EM. Development of a metric for a day of manageable pain control: derivation of pain severity cut-points for low back pain and osteoarthritis. Pain. 2003;106(1-2): $35-42$.

11. Conti PC, Pinto-Fiamengui LM, Cunha CO, Conti AC. Orofacial pain and temporomandibular disorders: the impact on oral health and quality of life. Braz Oral Res. 2012;26 Suppl 1:120-123.

12. Tölle T, Dukes E, Sadosky A. Patient burden of trigeminal neuralgia: results from a cross-sectional survey of health state impairment and treatment patterns in six European countries. Pain Pract. 2006;6(3): $153-160$.

13. Pigg M, Svensson P, Drangsholt M, List T. Seven-year follow-up of patients diagnosed with atypical odontalgia: a prospective study. J Orofac Pain. 2013;27(2):151-164.

14. Zigmond AS, Snaith RP. The Hospital Anxiety and Depression Scale. Acta Psychiatr Scand. 1983;67(6):361-370.

15. Headache Classification Subcommittee of the International Headache Society. The International Classification of Headache Disorders: 2nd edition. Cephalalgia. 2004;24 Suppl 1:9-160.

16. Van Grootel RJ, van der Bilt A, van der Glas HW. Long-term reliable change of pain scores in individual myogenous TMD patients. Eur J Pain. 2007;11(6):635-643.

17. Hirschfeld G, Zernikow B. Variability of "optimal" cut points for mild, moderate, and severe pain: neglected problems when comparing groups. Pain. 2013;154(1):154-159.

18. Cairns BE. The influence of gender and sex steroids on craniofacial nociception. Headache. 2007;47(2):319-324.

19. Paller CJ, Campbell CM, Edwards RR, Dobs AS. Sex-based differences in pain perception and treatment. Pain Med. 2009;10(2):289-299.

20. Carlsson GE, Ekbäck G, Johansson A, Ordell S, Unell L. Is there a trend of decreasing prevalence of TMD-related symptoms with ageing among the elderly? Acta Odontol Scand. 2014;72(8):714-720.

21. Johansson A, Unell L, Carlsson GE, Söderfeldt B, Halling A. Risk factors associated with symptoms of temporomandibular disorders in a population of 50- and 60-year-old subjects. J Oral Rehabil. 2006;33(7): 473-481.

22. Komiyama O, Obara R, Iida T, et al. Age-related associations between psychological characteristics and pain intensity among Japanese patients with temporomandibular disorder. J Oral Sci. 2014;56(3): 221-225.

23. Gerrits MM, van Oppen P, van Marwijk HW, Penninx BW, van der Horst HE. Pain and the onset of depressive and anxiety disorders. Pain. 2014;155(1):53-59.

24. De Heer EW, Gerrits MM, Beekman AT, et al. The association of depression and anxiety with pain: a study from NESDA. PLoS One. 2014;9(10):e106907.

25. Gerrits MM, Vogelzangs N, van Oppen P, van Marwijk HW, van der Horst H, Penninx BW. Impact of pain on the course of depressive and anxiety disorders. Pain. 2012;153(2):429-436.

26. McCracken LM. Learning to live with the pain: acceptance of pain predicts adjustment in persons with chronic pain. Pain. 1998;74(1): 21-27.

27. McCracken LM, Eccleston C. Coping or acceptance: what to do about chronic pain? Pain. 2003;105(1-2):197-204.

28. McCracken LM, Vowles KE, Eccleston C. Acceptance of chronic pain: component analysis and a revised assessment method. Pain. 2004;107(1-2):159-166.

29. Shacham S. A shortened version of the Profile of Mood States. J Pers Assess. 1983;47(3):305-306.

30. Zakrzewska JM, Linskey ME. Trigeminal neuralgia. BMJ. 2014; 348:g474. 
31. Komiyama O, Wang K, Svensson P, Arendt-Nielsen L, Kawara M, De Laat A. The influence of psychological state on the masseteric exteroceptive suppression reflex and somatosensory function. Clin Neurophysiol. 2008;119(10):2321-2328.

32. Komiyama O, Obara R, Uchida T, et al. Pain intensity and psychosocial characteristics of patients with burning mouth syndrome and trigeminal neuralgia. J Oral Sci. 2012;54(4):321-327.

33. Wong WS, Chen PP, Yap J, Mak KH, Tam BK, Fielding R. Assessing depression in patients with chronic pain: a comparison of three rating scales. J Affect Disord. 2011;133(1-2):179-187.

34. Yap AU, Tan KB, Chua EK, Tan HH. Depression and somatization in patients with temporomandibular disorders. J Prosthet Dent. 2002;88(5):479-484.

35. Yap AU, Chua EK, Hoe JK. Clinical TMD, pain-related disability and psychological status of TMD patients. J Oral Rehabil. 2002;29(4): 374-380.

36. Yap AU, Dworkin SF, Chua EK, List T, Tan KB, Tan HH. Prevalence of temporomandibular disorder subtypes, psychologic distress, and psychosocial dysfunction in Asian patients. J Orofac Pain. 2003;17(1): 21-28.
37. Thorne FM, Morley S. Prospective judgments of acceptable outcomes for pain, interference and activity: patient-determined outcome criteria. Pain. 2009;144(3):262-269.

38. Farrar JT, Young JP, LaMoreaux L, Werth JL, Poole RM. Clinical importance of changes in chronic pain intensity measured on an 11-point numerical pain rating scale. Pain. 2001;94(2):149-158.

39. Emshoff R, Emshoff I, Bertram S. Estimation of clinically important change for visual analog scales measuring chronic temporomandibular disorder pain. J Orofac Pain. 2010;24(3):262-269.

40. Dworkin RH, Turk DC, Farrar JT, et al. Core outcome measures for chronic pain clinical trials: IMMPACT recommendations. Pain. 2005;113(1-2):9-19.

41. Zakrzewska JM, Jassim S, Bulman JS. A prospective, longitudinal study on patients with trigeminal neuralgia who underwent radiofrequency thermocoagulation of the gasserian ganglion. Pain. 1999;79(1): $51-58$.

42. Lee JY, Chen HI, Urban C, et al. Development of and psychometric testing for the Brief Pain Inventory-Facial in patients with facial pain syndromes. J Neurosurg. 2010;113(3):516-523.
Journal of Pain Research

\section{Publish your work in this journal}

The Journal of Pain Research is an international, peer-reviewed, open access, online journal that welcomes laboratory and clinical findings in the fields of pain research and the prevention and management of pain. Original research, reviews, symposium reports, hypothesis formation and commentaries are all considered for publication.

\section{Dovepress}

The manuscript management system is completely online and includes a very quick and fair peer-review system, which is all easy to use. Visit http://www.dovepress.com/testimonials.php to read real quotes from published authors. 